\title{
A Survey on Data Mining Techniques in Image Mining
}

\author{
Varsha $\mathrm{N}^{*}$ \\ PES College of Engineering
}

DOI: https://doi.org/10.21467/proceedings.1.47

* Corresponding author email: nvarsha453@gmail.com

\begin{abstract}
Data mining is the search of large datasets to extract hidden and previously unknown patterns. Here image mining Extremely is focused on extracting implicit knowledge or patterns which are not explicity found in the images from collection of images or databases. This is the process of finding necessary information and knowledge in huge volume of data. Image mining is much more necessary for the efficient extraction of features in huge amount of complex data. It is a challenging field which extends traditional data mining focused from structured data to unstructured data such as image data. This paper presents a survey on various feature extractions in image mining.

Keywords-Image mining, Data, analyzing
\end{abstract}

\section{Introduction}

Mining is a process for discover the knowledge or check the pattern of the previous data. Once the patterns are found then these patterns are helps to make the decisions for the future strategies. sorting the information and getting specific information from databases has become of major significance. There is an enormous volume of information out there for individual due to huge analysis and development in the recent years.

In Image process, feature extraction starts from an initial set of measured information and builds derived values Pictures have visual character, they will be pictured in numerical kind, but great amount of numbers is to be evaluated so as to go looking image databases. Finding, extracting and classifying objects from pictures are the fundamental needs of process a picture with success. These as a novel class of information disagree from text data in many aspects as in terms of their nature thus in terms of storing and retrieving. Looking out info at intervals pictures represents a special entity of information process. Figure 1 shows the schematic illustration of image mining method.

(C) 2018 Copyright held by the author(s). Published by AIJR Publisher in Proceedings of the $3^{\text {rd }}$ National Conference on Image Processing, Computing, Communication, Networking and Data Analytics (NCICCNDA 2018), April 28, 2018.

This is an open access article under Creative Commons Attribution-NonCommercial 4.0 International (CC BY-NC 4.0) license, which permits any non-commercial use, distribution, adaptation, and reproduction in any medium, as long as the original work is properly cited. ISBN: $978-81-936820-0-5$ 


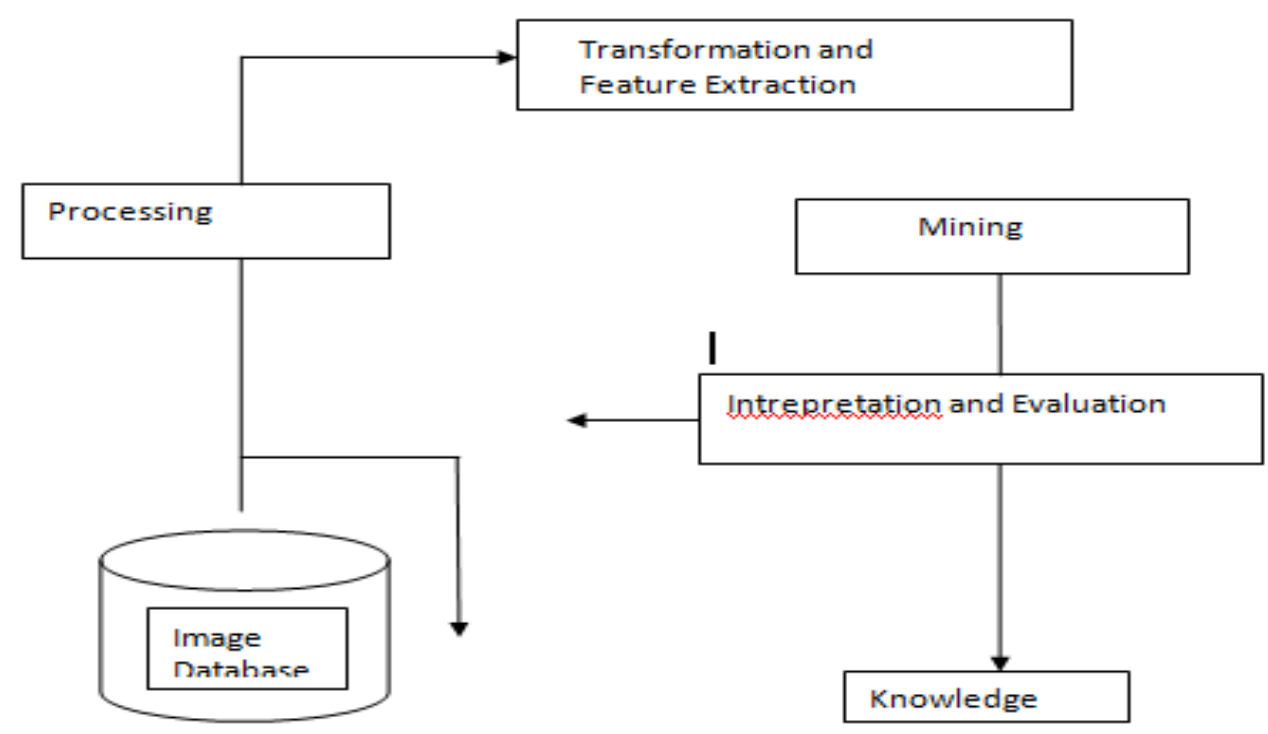

Fig. 1: General Image Mining system

\section{FEATURES}

\subsection{OBJECT BASED FEATURE EXTRACTION}

Feature extraction is said to spatiality reduction. The object-based feature extraction is predicated on single-object characterization and multiobject spatial relationships. The system extracts objects from the image content victimization the differential morphological profiles (DMP). These objects are one by one processed to extract form and spectral options. In Image process, feature Extraction starts from an initial set of measured information and builds derived values. The features used were the following.

\subsubsection{Color Moments}

For the color moments mean, variance, and skewness of the HSV values were calculated and stored in a 9-D feature. The RGB colour coordinates of the pixels were first transformed to the hue-saturation-value (HSV) coordinate system.

\subsubsection{Texture}

N. Boujemaa and J. Fauqueur (2001) says Visual appearance is automatically measured by numerical signature of image features such as color, texture, shape, or most often a combination of them. More specific image signatures have to be developed for special content and situations. Then, M. Datcu et al., (2003) proposed a system that extracts structural information from remote sensing images by selecting those prior models that best explain the

Proceedings of the $3^{\text {rd }}$ National Conference on Image Processing, Computing, Communication, Networking and Data Analytics (NCICCNDA 2018) 
A Survey on Data Mining Techniques in Image Mining

structures within an image. On the lowest level, the image data, is used to capture the spatial, spectral, and geometric structures in the image.

S. Durbha and R. King (2005), used the primitive feature extraction techniques. The primitive features that have been extracted from each region are used to perform an unsupervised classification (using KPCA), which extracts components of features that are nonlinearly related to the input variables. Since, the feature extraction produces large volumes of data that cannot be managed in practice. Later, Ashfaqur Rahman et al., (2016) computed three sets of features from the processed images of the rock surfaces.

\subsubsection{Color Features}

A histogram of the same size (i.e., number of bins) is computed from the three channels representing the distribution of different color components across the image.

Each color image is composed of three color channels: red (R), green $(G)$, and blue (B).

\subsubsection{Texture Features}

Images are first converted to gray level images to compute texture features. Gray Level Cooccurrence Matrix (GLCM) is a tabulation of how often different combinations of pixel brightness values (grey levels) occur in an image. The variation of gray level values (highs and lows) across the image is encoded in a GLCM.

\subsubsection{Edge Features}

Here the image is divided into $N \times N$ sub-images. Each sub image is further subdivided into $B \times B$ square blocks. An edge detection algorithm is applied on the gray level image to compute edges.

\subsection{General feature Extraction}

The overall Feature Extraction relies on spectral options and texture options. This is the extension of conventional linear discriminant analysis. Two nonlinear feature extraction algorithms based on this frame

\subsection{Tile-Based Feature Extraction}

The tile-based feature extraction is performed on the individual tiles. Each ingested multispectral image is subdivided into image tiles of size $256 \mathrm{~m} \times 256 \mathrm{~m}$.

\subsection{Anthropogenic Feature Extraction}

The phylogenesis feature extraction relies on linear options. Anthropogenic image content is often semisynthetic parts detectable within the satellite representational process like roads, buildings, and alternative structures. 


\section{CONCLUSION}

This paper presents a survey on data mining techniques for varied features that was planned earlier by researchers for the higher development within the field of image mining. Image mining handles with the hidden data extraction, image knowledge association and extra patterns that do not seem to be clearly accumulated within the pictures. Image mining is just a growth of information mining within the field of image process. Image mining handles with the hidden data extraction, image knowledge association and extra patterns that do not seem to be clearly accumulated within the pictures.

\section{REFERENCES}

[1] Kevin Alons, Mihai Datcu. Accelerated probabilistic learning concept for mining heterogeneous earth observation images. IEEE Journal of Selected Topics in Applied Earth Observations and Remote Sens. 2015; 8(7): 3356- 3371p.

[2] Yong Rui et al. Image retrieval: Current techniques, promising directions, and open issues. Journal of Visual Communication and Image Representation. 1999; 10(1): 39-62p.

[3] D. Espinoza-Molina, M. Datcu. Earth-observation image retrieval based on content, semantics, and metadata. IEEE Trans. Geosci. Remote Sens. 2013; 51(11): 5145-5159p.

[4] C. Bizer, T. Heath, T. Berners-Lee. Linked data-The story so far. Int. J. Semant. Web Inf. Syst. 2009; 5(3): 1- 22p.

[5] C.-R. Shyu, M. Klaric, G. J. Scott, et al. GeoIRIS: Geospatial information retrieval and indexing system - Content mining, semantics modeling, and complex queries. IEEE Trans. Geosci. Remote Sens. 2007; 45(4): 839-852p

[6] M. Molinier, J. Laaksonen, T. Hame. Detecting man-made structures and changes in satellite imagery with a contentbased information retrieval system built on self-organizing maps. IEEE Trans. Geosci. Remote Sens. 2007; 45(4): 861$874 \mathrm{p}$.

[7] M. Datcu et al. Information mining in remote sensing image archives: System concepts. IEEE Trans. Geosci. Remote Sens. 2003; 41(12): 2923-2936p.

[8] S. Durbha, R. King. Semantics-enabled framework for knowledge discovery from earth observation data archives. IEEE Trans. Geosci. Remo Sens. 2005; 43(11): 2563-2572p.

[9] N. Boujemaa, J. Fauqueur. Ikona: Interactive specific and generic image retrieval. In Proc. Int. Workshop Multimedia Content Based Indexing Retrieval. 2001; 2-5p

[10] Ramadass Sudhir. A Survey on imag mining techniques: Theory and applications. In Computer Engineering and Intelligent Systems 2011; 44-53p

[11]Jie Chen. Ikona WLD: A robust local image descriptor. IEEE Transactions on Pattern Analysis and Machine Intelligence. 2010; 32(9): 1705- 1720p.

[12]T.Gladina Nisia, S Rajesh: survey on selection of features for content based image retrieval in image mining

[13]Barbora Zahradnikova Sona, Duchovicova, Peter Schreiber. Image mining: Review and new challenge(IJASA) International journal of Advance Computer Science and Apllications. 2015; 6(7);242-246p

[14]S. C. D. Pinto et al. 3D facial expression analysis by using $2 \mathrm{~d}$ and $3 \mathrm{~d}$ wavelet transforms. IEEE International Conference on Image processing,2011;1293-1297p 